\title{
A rare case of Klebsiella pneumoniae myocardial abscess
}

\author{
Mohammad Abdul-Waheed, MD, ${ }^{\text {a }}$ Mian Atif Yousuf, MD, ${ }^{\mathrm{b}}$ Eric W. Schneeberger, MD ${ }^{\mathrm{c}}$ \\ Tehmina Naz, MD, FACC, ${ }^{\mathrm{a}}$ Daniel C. Eckert, MD, ${ }^{\mathrm{a}}$ Ginger Conway, RN, CNP, ${ }^{\mathrm{a}}$ and Tarek Helmy, MD, \\ FACC, ${ }^{\text {a }}$ Cincinnati, Ohio
}

Myocardial abscess of the left ventricular free wall in the absence of infective endocarditis (IE) is very unusual. Most cases are discovered during autopsies and are due to Gram-positive cocci. ${ }^{1,2}$ We present a rare case of Klebsiella pneumoniae causing myocardial abscess of the left ventricular free wall. The patient had no evidence of valvular endocarditis or bacteremia, and the abscess was discovered during coronary artery bypass grafting surgery (CABG).

\section{CLINICAL SUMMARY}

A 58-year-old obese woman with history of diabetes mellitus and coronary artery disease presented to the outpatient clinic with a 2-day history of left-sided chest discomfort and associated nausea. It was constant and did not change with posture or inspiration. She denied any shortness of breath, cough, fevers, chills, or night sweats. Physical exam was unremarkable, and a white blood cell count was within normal limits. An electrocardiogram revealed mild ST elevations in inferior leads, and she was promptly sent to the cardiac catheterization laboratory where she was found to have triple-vessel coronary disease and was referred for CABG. During the surgery, attempts at freeing the adherent anterior pericardium from the left ventricular free wall exposed a $5 \times 3-\mathrm{cm}$ myocardial abscess that was $2 \mathrm{~mm}$ deep (Figure 1). The abscess exuded frank pus. There was a focal area within the abscess that was $4 \mathrm{~mm}$ deep and was $2.5 \times 1 \mathrm{~cm}$ in dimension. The abscess was located just medial to the first diagonal branch of the left anterior descending artery. An intraoperative epicardial ultrasound revealed a $10-\mathrm{mm}$-thick ventricular wall with the superficial abscess measuring $4 \mathrm{~mm}$ at its deepest point (Figure 2). Once the pus was completely expressed, the abscess crater and pericardium were thoroughly irrigated with warm saline. Gram staining of the pus revealed Gram-negative rods. The decision was made to proceed with bypassing the coronary arteries that were away from the region of the abscess. The patient thus had a left internal mammary artery graft to the mid

\footnotetext{
From the Department of Medicine, Division of Cardiovascular Diseases, ${ }^{\text {a }}$ Department of Medicine, ${ }^{b}$ and Department of Cardiothoracic Surgery, ${ }^{\mathrm{c}}$ University of Cincinnati, Cincinnati, Ohio.

Disclosures: None.

Received for publication March 31, 2008; accepted for publication July 6, 2008; available ahead of print Feb 23, 2009.

Address for reprints: Mohammad Abdul Waheed, MD, University of Cincinnati, Department of Medicine, Division of Cardiovascular Diseases, 231 Albert Sabin

Way, Cincinnati, OH (E-mail: abdulwm@ucmail.uc.edu).

J Thorac Cardiovasc Surg 2010;139:e34-5

$0022-5223 / \$ 36.00$

Copyright (c) 2010 by The American Association for Thoracic Surgery doi:10.1016/j.jtcvs.2008.07.056
}

left anterior descending artery and a reverse saphenous vein graft to the distal right coronary artery. The patient had chest tubes placed in the left pleural space, pericardial space, and anterior mediastinum. The medial sternotomy was then closed in layers. The patient received intraoperative gentamycin intravenously, and a dilute solution of gentamycin was introduced through the mediastinal chest tube. Her initial postoperative period was complicated with hypotension from myocardial dysfunction (echocardiogram showed left ventricular ejection fraction of $20 \%$ and diastolic dysfunction) requiring multiple vasopressors and associated renal failure requiring continuous venovenous hemodialysis. Cultures from the abscess grew $K$ pneumoniae. A transesophageal echocardiogram, white blood cell nuclear scan, and blood cultures to locate a source of infection were unyielding. The patient could not get a contrast computed tomography scan secondary to renal failure and was above the permissible weight limit for a magnetic resonance imaging scan. She was treated with aztreonam and ciprofloxacin for 6 weeks, and her kidney function gradually normalized. She was then transferred to a physical rehabilitation facility where she recovered completely and was discharged home in stable condition.

\section{DISCUSSION}

Myocardial abscesses are seldom diagnosed in living subjects by noninvasive tests and might occasionally be discovered

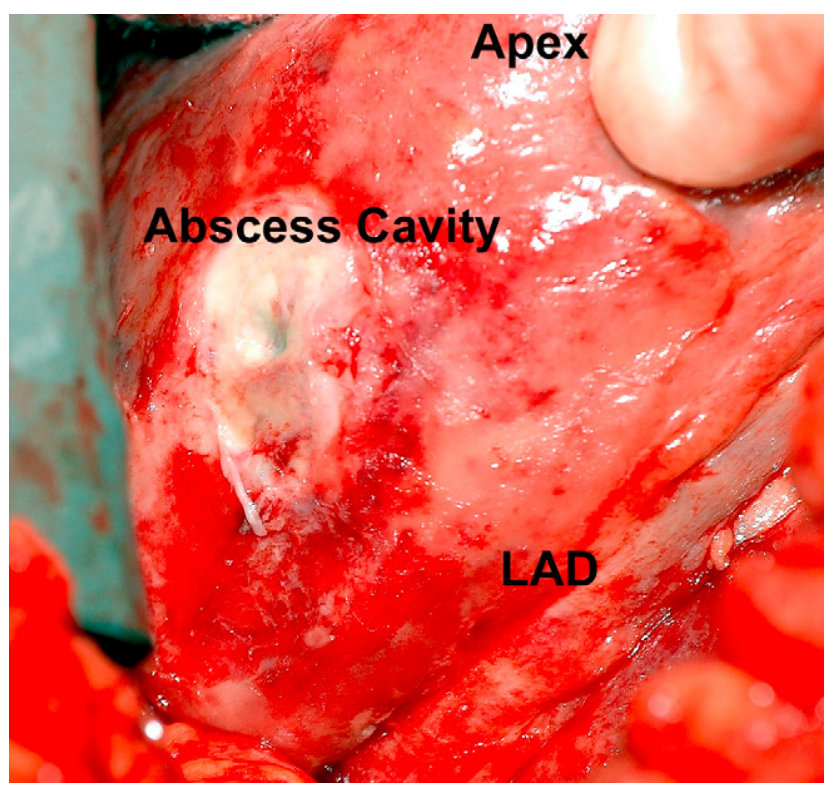

FIGURE 1. Intraoperative picture of the left ventricular free wall abscess. 


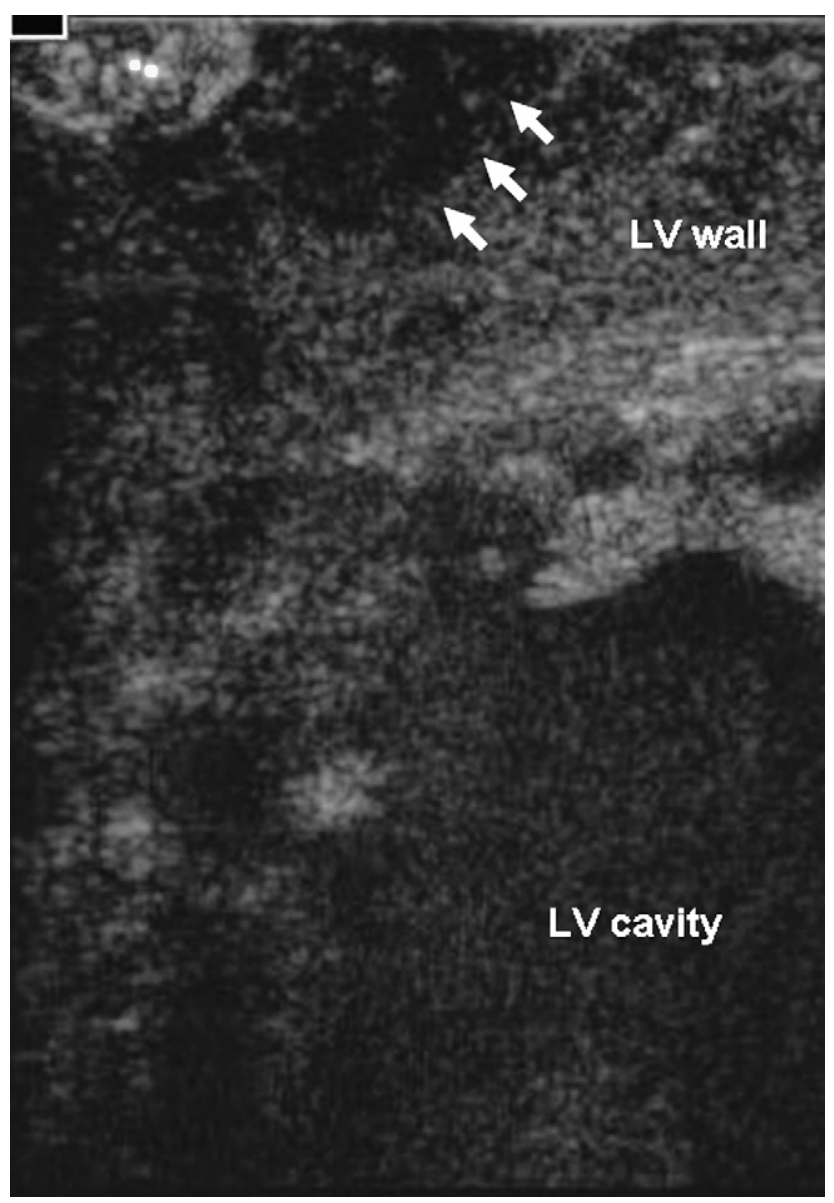

FIGURE 2. Arrows indicate the abscess in the anterior left ventricular wall on epicardial echocardiography.

during cardiac surgery or autopsy. ${ }^{3}$ The reported incidence during autopsy ranges between $0.2 \%$ and $1.5 \% .^{1,2}$ They usually occur in the setting of IE with Gram-positive cocci ${ }^{1,2}$ and some- times in infarcted myocardium when circulating microorganisms get seeded in the region. ${ }^{4}$ Our patient, who presented with chest pain and electrocardiogram changes consistent with acute coronary syndrome (ACS), was incidentally found to have $K$ pneumoniae myocardial abscess during CABG.

Maslow et $\mathrm{al}^{4}$ have reported the only other case of myocardial abscess due to $K$ pneumoniae. Their patient had $K$ pneumoniae urosepsis associated with acute myocardial infarction, resulting in myocardial abscess and papillary muscle rupture. The diagnosis was made during surgery for mitral valve replacement. The patient improved after therapy with cefotaxime; however, cardiac rupture occurred on postoperative day 6 .

There are no guidelines for treating $K$ pneumoniae infection involving the heart but a 6 -week course of aztreonam and ciprofloxacin proved effective in our patient. She is still doing well at the end of 1 year. To the best of our knowledge, this patient is the only documented survivor of a $K$ pneumoniae myocardial free wall abscess. A cardiac computed tomography or magnetic resonance imaging scan for patients with high clinical suspicion of a cardiac infection is a good test for diagnosing as well as localizing a myocardial free wall abscess. This might increase the incidence of detecting myocardial abscesses in living subjects, rather than it being predominantly an autopsy finding.

\section{References}

1. Flaxman N. Myocardial abscess. JAMA. 1943;122:804.

2. Sanson J, Slodki S, Gruhn JG. Myocardial abscesses. Am Heart J. 1963; 66:308-8.

3. Rohmann S, Seifert T, Erbel R, et al. Identification of abscess formation in nativevalve infective endocarditis using transesophageal echocardiography: implications for surgical treatment. Thorac Cardiovasc Surg. 1991;39:273-80.

4. Maslow MJ, Reitano JM, Schnall HA, Shah AT, Simberkoff MS, Rahal JJ. Myocardial abscess due to Klebsiella pneumoniae complicating acute infarction. Am J Med Sci. 1984;287:58-60.

\section{Sex reversal and hypoplastic left heart syndrome}

\author{
Andrew M. Atz, MD, ${ }^{\text {a }}$ Charleston, SC
}

Michael J. Walsh, MD, ${ }^{\mathrm{a}}$ Elizabeth T. Walsh, MD, ${ }^{\mathrm{b}}$ Yaw Appiagyei-Dankah, MD, ${ }^{\mathrm{b}}$ and
The prognosis of congenital heart disease, especially in its most complex forms, is complicated when associated with extracardiac anomalies. Meacham syndrome, or PAGOD syndrome (pulmonary tract and pulmonary artery hypoplasia, agonadism, omphalocele, diaphragmatic defect, and dextrocardia) is a constellation of genital, cardiac, and pulmonary malformations that carries a poor prognosis. We report the case of a patient with sex reversal and hypoplastic left heart syndrome without congenital diaphragmatic hernia who has survived to Fontan completion and is doing well. 